\title{
I nuovi LEA: si chiude un ciclo e si apre una sfida per il SSN
}

\author{
di Elio Borgonovi
}

Va dato atto al Governo di aver chiuso un ciclo durato quindici anni con l'approvazione dei nuovi LEA. Le Regioni, le Aziende Sanitarie, gli operatori e soprattutto i cittadini attendevano un aggiornamento di quanto era stato definito nel 2001 che ogni anno diventava sempre più necessario e urgente in rapporto all'evoluzione dei bisogni, delle conoscenze nel campo della tutela della salute, dell'innovazione tecnica, tecnologica e dei modelli assistenziali.

I punti centrali del provvedimento, che sono stati evidenziati con maggiore o minore enfasi sono riassumibili nei seguenti termini:

- piano di vaccini garantiti gratuitamente alla popolazione per rafforzare l'aspetto preventivo anche di fronte a situazioni che hanno allarmato l'opinione pubblica (es. casi di meningite, influenza che quest'anno ha colpito in modo pesante la popolazione, campagne contro le vaccinazioni dei bambini);

- inserimento di nuove prestazioni rese possibili dal progresso scientifico;

- aggiornamento dei setting assistenziali nel quali le prestazioni possono essere erogate quali day surgery, altre prestazioni in day hospital, prestazioni specialistiche ambulatoriali, migliore qualificazione delle condizioni di cronicità e multicronicità;

- aumento del numero delle malattie rare anche se le organizzazioni e le federazioni che le raggruppano hanno evidenziato vari aspetti di criticità e di perplessità destati dalla mancata inclusione di altre patologie;

- aggiornamento delle tecnologie, delle protesi e in generale degli ausili che consentono risposte più appropriate e di qualità più elevata.

L'approvazione del nuovo Decreto LEA deve essere considerata anche l'occasione per riportare l'attenzione dei vari soggetti pubblici e privati e dell'opinione pubblica sul tema cruciale della divaricazione che si è manifestata negli ultimi quindici anni tra i livelli assistenziali effettivi garantiti dalle Regioni. È ovvio che non basta un decreto per invertire una tendenza strutturale, tema su cui si ritorna più avanti, ma sicuramente l'approvazione dei nuovi LEA costituisce una occasione per riflettere su un tema che ogni anno è diventato più critico e che, se non affrontato adeguatamente a livello statale, delle Regioni, della Conferenza Stato-Regioni, potrebbe dar luogo a problemi di sostenibilità sociale che non sono meno rilevanti di quelli della sostenibilità economica. Va riconosciuto che le critiche in questo caso sono state minori che per altri provvedimenti, non è dato sapere se per la soddisfazione connessa alla conclusione di un processo durato molti anni, per la speranza riposta nella capacità del sistema di recuperare almeno in parte il terreno perduto, o perché il Paese è alle prese con molti problemi, compreso quello delle drammatiche calamità naturali. Alcune di queste critiche pur comprensibili e a volte anche condivisibili, sono conseguenza inevitabile del lungo iter legislativo articolato su una fase tecnica relativa alla individuazione dei nuovi LEA, il coinvolgimento delle Regioni imposto dal fatto che si tratta di una materia concorrente tra Stato e Regioni, situazione che si intendeva semplificare con la riforma costituzionale bocciata dal referendum del 4 dicembre 2016, il confronto con il Parlamento, la 
verifica delle compatibilità finanziarie. Un iter che è stato ulteriormente penalizzato dal cambio dei governi a partire dal 2011 e dalla rigidità dei processi legislativi. Infatti, i provvedimenti non possono essere modificati durante l'iter salvo ricominciare le consultazioni e $i$ vari passaggi, e quindi è per certi aspetti inevitabile e strutturale il fatto che alcuni LEA e alcune tecnologie appaiano già in parte obsolete nel momento in cui si è arrivati all'approvazione. L'altra componente delle critiche deriva dalla sottovalutazione di una condizione strutturale non eliminabile, cioè quella della divaricazione tra attese e aspettative di vari gruppi sociali (es. i rappresentanti di patologie rare) e vincoli finanziari e di capacità reale di risposta da parte del sistema. Si tenga conto che le malattie rare sono oltre 7000 e che lo stesso concetto di malattia rara può essere diversamente interpretato. Inoltre, sia per le malattie rare sia per le cronicità, $i$ tecnici e i decisori politici a livello regionale e nazionale hanno dovuto considerare l'impatto in termini di costo più o meno elevato e di sostenibilità da parte del SSN da un lato e delle famiglie dall'altro.

Ogni legge o provvedimento formale rappresenta da un lato la conclusione di un iter più o meno lungo, dall'altro l'apertura di una fase di cambiamento che deve essere proiettata nel futuro. L'approvazione dei nuovi LEA apre una fase di nuove sfide per il SSN, alle quali occorre prepararsi da subito. Innanzitutto il già ricordato tema del finanziamento, in quanto esiste la certezza che l'entità del Fondo Sanitario Nazionale non potrà aumentare in modo significativo né nel prossimo decennio, né in termini assoluti o come percentuale sul PIL. L'Italia è alle prese con un quadro internazionale destinato a rendere ancora più stringenti i vincoli di finanza pubblica. Ciò costringerà le Regioni ad applicare concretamente il principio di "fare più (i nuovi LEA e gli aggiornamenti che dovranno seguire) con meno o con risorse inalterate in termini reali (finanziamento dello Stato e da parte delle Regioni)". Una sfida che dura da almeno vent'anni ma che dovrà essere affrontata aumentando la velocità dei processi di riallocazione di risorse. Purtroppo da alcuni anni, soprattutto dopo la crisi 2007/2008, si è verificato un fenomeno "semantico" in quanto sono state fatte dichiarazioni sulla necessità di razionalizzazione mentre nel concreto vi è stato un razionamento delle risorse rispetto ai bisogni. Si potrebbe dire che si scrive "razionalizzazione" ma si legge "razionamento", implicito e determinato dalle scelte quotidiane. Non è più possibile "navigare a vista" né per la finanza pubblica nel suo complesso né per il finanziamento del SSN. Ė necessario lanciare un progetto di sensibilizzazione che si richiami al principio della "distruzione creatrice" cosi cara agli economisti dalla formulazione di J. Schumpeter. Un progetto che sia fondato su tre pilastri:

- definizione di una solida e innovativa teoria di riferimento sui criteri di allocazione di risorse finanziarie dallo Stato alle Regioni e dalle Regioni alle Asl, che sia focalizzata sul concetto di performance declinato per livelli istituzionali di responsabilità all'interno delle Aziende Sanitarie Territoriali (AST) e Aziende Ospedaliere (AO), per cluster di pazienti;

- raccolta sistematica di buone pratiche di spending review "realistica", sia a livello nazionale sia a livello internazionale, basata sulla logica dei "tagli" (delle attività e delle spese inutili o a bassa utilità in termini di qualità dell'assistenza) e "investimenti" (intesi in senso lato come spese con più elevata utilità in termini di qualità dell'assistenza);

- azioni per lo sviluppo, rafforzamento, consolidamento e diffusione delle conoscenze e competenze necessarie per dare efficacia a interventi di riqualificazione/razionalizzazione, poiché sono le persone che consentono di dare concretezza alle teorie, alle politiche.

I nuovi livelli di assistenza si inseriscono in un contesto completamente diverso da quello del 2001, in quanto vi è stata un'evoluzione sul piano teorico, delle conoscenze pratiche e delle sperimentazioni che hanno portato all'affermazione del concetto di presa in carico del paziente. Come è noto, il concetto di Livelli Essenziali di Assistenza si collega prevalentemente, anche se non esclusivamente, a quello di prestazioni e a sua volta a quello di DRG. Va detto che semanticamente il concetto di LEA dovrebbe, si sottolinea il condizionale, essere collegato al risultato finale in termini di beneficio per i pazienti, ossia di recupero, mantenimento e promozione dello stato di salute. Tuttavia, la traduzione concreta fino ad alcuni anni fa è stata quella di prestazioni garantite. Per questo la definizione dei livelli essenziali del 2001 era focalizzata su interventi di tutela della salute, facilmente declinabili in termini di prestazioni o di setting assistenziali ben definiti: ricovero, day 
hospital, ambulatori. In quindici anni vi è stata una evoluzione delle conoscenze e della pratica che ha portato a distinguere tra output, prestazioni, risultati intermedi e outcome, soddisfazione dei bisogni, qualità dell'assistenza.

Questa evoluzione è stata solo in parte recepita nel nuovo schema di LEA, ma non è ancora riuscita a recepire in pieno il passaggio dal concetto di diagnosi e cura (cure) a quello di presa in carico del paziente (care). Un'evoluzione che si è consolidata per quanto riguarda le situazioni di cronicità, ma che riguarda anche le condizioni acute. È di più immediata comprensione il fatto che i pazienti cronici richiedano una molteplicità di prestazioni (somministrazione di farmaci, controlli periodici, eventuali ricoveri in strutture di assistenza a bassa complessità in caso di aggravamento delle condizioni) e gli erogatori, che sono responsabilizzati per la presa in carico dei pazienti, siano indotti a evitare prestazioni inappropriate e a garantire prestazioni efficaci non solo per una migliore soddisfazione dei pazienti, ma anche per la riduzione dei costi. La Regione Lombardia, prima tra le regioni italiane e forse anche a livello internazionale, il 30 gennaio 2017 ha approvato un'innovativa delibera riguardante il finanziamento di pazienti cronici basato non su DRG ma su tariffe complessive per la presa in carico. Tuttavia, anche i pazienti che si sottopongono a visite specialistiche o richiedono ricoveri per condizioni acute presentano situazioni che richiedono un approccio interdisciplinare. Per garantire nel migliore dei modi i LEA di tipo acuto è sempre più frequentemente necessario un approccio complessivo al paziente. Ciò, ancora una volta, per garantire una migliore qualità della risposta, un più elevato livello di soddisfazione, un recupero più rapido delle condizioni di benessere, ma anche una riduzione dei costi complessivi dell'assistenza. Ne consegue che la sostenibilità dei nuovi LEA può essere perseguita con una più elevata probabilità di successo, o se si vuole con un minore livello di insoddisfazione e criticità, solo se le Aziende Sanitarie Territoriali e le Aziende Ospedaliere saranno in grado di evolvere velocemente da un modello di prevenzione, diagnosi e cura, riabilitazione, trattamento delle cronicità basato su erogazione di prestazioni tra loro separate a un modello di presa in carico del paziente nel quale le prestazioni sono considerate in modo organico e sistemico, sia sullo stato di salute sia sul piano economico. Spetta a tutti raccogliere la sfida lanciata.

Il passaggio da un modello basato sulle prestazioni a un modello basato sulla presa in carico richiede una condizione essenziale, quella di introdurre con decisione sistemi evoluti di performance management. Si può dire che la logica delle valutazione delle performance sia presente da tempo nei sistemi di tutela della salute, con riferimento ai processi assistenziali. Infatti, può essere considerata tale la logica della evidence based medicine, che si fonda su trial clinici randomizzati che consentono di confrontare le performance di gruppi di pazienti sottoposti o non sottoposti a certi trattamenti o sottoposti a trattamenti differenziati (es. con farmaci già da tempo ritenuti efficaci e nuovi farmaci, device già sperimentati e nuovi device ecc.). Inoltre, indicatori di tipo epidemiologico su popolazioni o gruppi di pazienti, nonché l'analisi delle variazioni nel tempo o di differenze tra realtà simili, sono sistemi di performance. Meno usati e meno diffusi sono gli strumenti di performance management di tipo aziendale. In effetti già dagli inizi degli anni Novanta, a seguito dei decreti 502/92 e 517/93 che hanno realizzato il passaggio dalle USL alle ASL, caratterizzate dalla logica aziendale, l'introduzione dei sistemi di controllo e di gestione ha favorito la diffusione di indicatori di performance di efficacia, efficienza, economicità. In seguito, con la realizzazione del Piano Nazionale Esiti da parte di Agenas e con l'introduzione del DM 70/2015 vi è stata anche una ulteriore focalizzazione sul concetto e sul concetto e sugli indicatori di efficacia e di appropriatezza. Tuttavia è difficile contestare il fatto che l'evoluzione dell'ultimo quarto di secolo ha presentato due elementi di debolezza. Da un lato, vi è stata maggiore attenzione alla rilevazione dei dati e minore attenzione sulla loro utilizzazione, non vi è stato un adeguato passaggio dalla semplice misurazione (performance measurement) alla analisi, interpretazione, valutazione e decisione sulla base di indicatori di performance (performance management). Dall'altro lato, sono prevalsi approcci settoriali e vi sono state poche esperienze di tipo sistemico. Per questo l'approvazione dei nuovi LEA pone anche la sfida del rafforzamento di una cultura delle performance che Agenas intende accettare sulla base di una azione di sistema, articolata su questi ambiti:

- performance relative ai processi di prevenzione primaria e secondaria;

- performance dei processi di acquisto dei beni strumentali, distinguendo le performance relative ai prezzi di acquisto e ai volumi; 
- performance dei processi di acquisto all'esterno di prestazioni sanitarie per conto del SSN nella componente di prezzi, appropriatezza, qualità delle prestazioni, soddisfazione dei pazienti;

- performance dei processi assistenziali per acuti e pazienti urgenti non prevedibili, per esempio dei Pronto Soccorso e di altre strutture di urgenza;

- performance dei processi assistenziali con degenza programmabili, nelle diverse dimensioni di appropriatezza, qualità dei servizi, efficienza nel rapporto prestazioni (output)-fattori produttivi (input), costi (unitari e complessivi);

- performance dei processi di assistenza specialistica senza ricovero (day hospital, attività ambulatoriale ecc.);

- performance delle strutture per le cure intermedie;

- performance dei processi assistenziali connessi a cronicità, disabilità, non autosufficienza (presa in carico dei pazienti);

- performance relative ai processi di erogazione, in particolare valutazione degli esiti e della soddisfazione dei pazienti.

Come si è detto, su questo fronte si è scritto tanto e si è fatto tanto in Italia e all'estero, ma la sfida che viene posta dall'approvazione dei nuovi LEA è quella di creare un "sistema organico" di performance che rafforzi le due linee di influenza. Da un lato, quella che collega gli indirizzi nazionali alle scelte delle Regioni, al funzionamento delle AST $e$ AO. Dall'altro, quella che partendo da esperienze significative e innovative (le good practice) di alcune AST e AO possa diffondersi rapidamente a molte altre realtà, generando una linfa vitale per il miglioramento. Tra l'altro l'attenzione sulle performance e un loro uso più appropriato e sistemico potrà aiutare (forse) a intervenire anche sull'annoso e sempre più grave problema della differenziazione e divaricazione dei livelli essenziali effettivamente garantiti dalle diverse regioni. Porre le AST e le AO di fronte a un sistema organico di performance che consenta comparazioni con altre può attivare quello "spirito di emulazione" che scatta in presenza di adeguati stimoli, siano essi costituiti da criteri di finanziamento incentivanti, formazione del personale, azioni sulle motivazioni intrinseche di chi svolge una professione a servizio di altri. L'esistenza di un progetto nazionale sul tema delle performance può stimolare le regioni che oggi figurano nella parte bassa delle classifiche sulla qualità dell'assistenza a recuperare terreno. Peraltro si ricorda che il riequilibrio tra regioni non potrà mai avvenire per legge o perché si modificano i criteri di finanziamento, ma solo se vi sarà una forte e consapevole azione di accompagnamento, come previsto dalle norme sui piani di rientro che si sono succedute dal 2006 e le più recenti norme su efficientamento e qualificazione delle AO previste dalla Legge di Stabilità 2016 e dalla Legge di Bilancio 2017.

Non ci si deve mai stancare di ricordare che per affrontare sfide difficili occorre passare dalle enunciazioni di esigenze, di principi, di teorie alla loro attuazione. Mai come di fronte alle nuove sfide del SSN è appropriato l'adagio popolare secondo cui "tra il dire e il fare c'è di mezzo il mare". Il mare è costituito dalla attivazione del circolo vizioso tra conoscenze su ciò che sta cambiando, volontà di cambiare, potere reale per governare il cambiamento. È il classico problema di knowledge management che dalle singole persone e dalle singole aziende deve diventare processo istituzionale che raccorda e coordina il livello del decisore dei LEA (lo Stato), quello della programmazione e organizzazione (le Regioni), quello degli erogatori pubblici o privati dei servizi e delle prestazioni finali. Un problema che deve essere affrontato tenendo conto di evitare alcune trappole. La prima riguarda l'effetto annuncio, che consiste nell'eccesso di ottimismo nella comunicazione. Dire che i vaccini saranno gratis, che i nuovi LEA consentiranno di migliorare la qualità dell'assistenza, che le Regioni saranno indotte, obbligate, responsabilizzate in modo diverso dal passato è buona cosa, ma non bisogna eccedere per non alzare troppo il livello delle attese rispetto alle possibilità reali di mantenere le promesse fatte. Il secondo rischio da evitare è quello del "cambiamento a salti". Già si è sottolineato che iLEA sono stati cambiati dopo 15 anni, nonostante nel 2001 fosse previsto un aggiornamento a tempi più stretti. Con la nuova norma è stata istituita la Commissione Scientifica che dovrà aggiornare annualmente i LEA, occorrerà garantirle le condizioni istituzionali, organizzative, di indipendenza, informative, economiche per poter svolgere realmente le proprie funzioni. Il terzo rischio è quello della de-responsabilizzazione. Lo Stato ha approvato i nuovi LEA, 
prevedendo uno stanziamento di 800 milioni destinati a questo scopo, le Regioni hanno già affermato che si tratta di uno stanziamento insufficiente, gli organi competenti si sono già mossi con le società scientifiche e con le rappresentanze delle Aziende sanitarie per individuare misure di contenimento della spesa (es. passaggio di alcune prestazioni dal regime di ricovero ordinario a quello di day hospital o ambulatoriale, a volte senza tenere conto della sicurezza del paziente o di complicanze che potrebbero determinarsi), il che rischia di attivare il circolo vizioso del rinvio di responsabilità tra $i$ due livelli di governo. A loro volta le Regioni, mentre da un lato affrontano il confronto con lo Stato, dall'altro hanno attivato e attiveranno misure sempre più rigorose nei confronti delle Aziende sanitarie pubbliche e private, per stimolare/imporre un recupero di efficienza. A loro volta gli erogatori pubblico-privati hanno già fatto sapere in forme diverse che di fronte a vincoli sempre più stringenti sarà difficile erogare i nuovi LEA secondo le indicazioni centrali e regionali. Per rompere il circolo vizioso della de-responsabilizzazione sarà necessario agire su raccolta tempestiva di dati e informazioni, definizione di performance, accettazione del confronto senza preconcetti, condivisione di soluzioni "realistiche".

In conclusione si può dire che la sfida posta dai LEA potrà essere affrontata con la ragionevole attesa di un miglioramento tramite azioni in grado di generare discontinuità nella cultura prevalente. Per essere più precisi occorrerà agire per:

- potenziare la cultura delle "scelte strategiche" in grado di superare la cultura della routine, della gestione quotidiana, della urgenza che diventa normalità;

- passare da una cultura del breve periodo a una di lungo periodo, ossia a una visione di lungo periodo e realizzazione con interventi di breve periodo tra loro coordinati;

- sostituire la logica di norme che devono essere applicate a quella di norme che sostituiscono obiettivi e responsabilizzano coloro che devono erogare i nuovi LEA (empowerment delle aziende che stanno alla base del sistema e, all'interno di esse, di coloro che operano a vari livelli di responsabilità);

- potenziare una formazione integrata di tipo formale, informale e non formale che, in sintesi, potremmo qualificare come integrazione tra processi di teaching (formazione di base all'università), learning (formazione in aula per executive, ossia per persone che già operano), learning by doing (ossia il processo di apprendimento sul campo).

Il futuro del SSN è nelle mani (abilità operative, es. chirurghi, infermieri ecc.), nelle teste (conoscenze e razionalità) e nel cuore (emozioni e passioni) di persone motivate a fare bene. Per alzare la motivazione di chi ogni giorno è alle prese con la salute di tutti noi occorre che ognuno di noi, sia chi è nella condizione di paziente sia chi è in altre funzioni (ricercatori, operatori dei media, manager e imprenditori che interagiscono con il SSN, assicurazioni sanitarie, fondi salute), diano messaggi equilibrati di critica al sistema (utili per stimolare il miglioramento) e fiducia (condizione necessaria per motivare le persone). Affermazioni del tipo "ottimismo della volontà e pessimismo della ragione" devono essere sostituite dalla ragionevole fiducia nella capacità delle persone di affrontare la sfida del cambiamento posta dai nuovi LEA. 